\title{
A RELAÇÃO ENTRE A POLUIÇÃO DO AR E AS PRINCIPAIS VARIÁVEIS CLIMATOLÓGICAS NO MUNICÍPIO DE CANOAS-RS
}

\author{
TIBULO, Cleiton - tibulo_cleiton@hotmail.com \\ Universidade Federal de Santa Maria / UFSM \\ ZANINI, Roselaine Ruviaro - rrzanini63@gmail.com \\ Universidade Federal de Santa Maria / UFSM
}
FERRAZ, Simone Erotildes Teleginski - simonetfe@gmail.com
Universidade Federal de Santa Maria / UFSM
BOIASKI, Nathalie Tissot - ntboiaski@gmail.com
Universidade Federal de Santa Maria / UFSM
TIBULO, Vanessa de Carli - vaneza_dc@yahoo.com.br Universidade Federal de Santa Maria / UFSM

\begin{abstract}
RESUMO: Nas últimas décadas, junto com o crescente aumento do número de veículos no Brasil, também tem crescido a preocupação com fatores ligados à poluição e suas consequências para a sociedade. Nesse contexto, o presente trabalho teve por objetivo aplicar técnicas estatísticas de análise multivariada aos dados de poluentes do ar e variáveis meteorológicas para investigar a relação entre estes componentes. Além disso, foram ajustados modelos de Previsão de Séries Temporais para os índices de poluição do ar, utilizando as variáveis climatológicas como exógenas aos modelos, com a finalidade de comparar o desempenho dos modelos. Os dados foram coletados junto ao Instituto Nacional de meteorologia (INMET) (variáveis climatológicas) e Fundação Estadual de Proteção Ambiental (FEPAM) (poluentes do Ar) referente ao município de Canoas-RS, durante $o$ ano de 2014. Os resultados da análise de cluster mostram a formação de dois grupos bem definidos: $O$ cluster 1 reúne as variáveis mais próximas $\left(\mathrm{O}_{3}\right.$, Insolação, Evaporação, Temperatura Mínima e Temperatura Máxima), o cluster 2, as variáveis mais próximas $\left(\mathrm{SO}_{2}, \mathrm{CO}, \mathrm{PM} 10\right)$. Ajustaram-se modelos de previsão de Séries Temporais para a variável $\mathrm{O}_{3}$, usando as demais variáveis do grupo 1 como exógenas. O modelo de Série Temporal que permite a inclusão de variáveis exógenas (SARIMAX), obteve melhores resultados de previsão em relação aos seus concorrentes (SARIMA e da classe de Alisamento Exponencial). Conclui-se que o uso conjunto de modelos de análise multivariada e séries temporais proporciona melhores resultados de previsão. Destaca-se a ampla possibilidade de utilização das técnicas conjuntas de análise multivariada e séries temporais, podendo ser utilizadas como ferramenta de apoio nas tomadas de decisão.
\end{abstract}

PALAVRAS-ChAVe: Poluição do ar. Variáveis climatológicas. Modelos de séries temporais. Modelos de análise multivariada

\section{RELATIONSHIP BETWEEN AIR POLLUTION AND MAIN CLIMATOLOGICAL VARIABLES IN CANOAS-RS}

ABSTRACT: In recent decades, along with the growing number of vehicles in Brazil, there has also been growing concern about pollution-related factors and their consequences for society. In this context, the objective of the present work was to apply statistical multivariate analysis techniques to air pollutant data and meteorological variables to investigate the relationship between these components. In addition, time series forecast models for air pollution indexes were adjusted, using the climatological variables as exogenous to the models, in order to compare the performance of the models. The data were collected from the National Institute of Meteorology (INMET) 
(climatological variables) and the State Foundation for Environmental Protection (FEPAM) (Air pollutants) referring to the city of Canoas-RS, during the year 2014. The results of the analysis of Cluster 1 shows the formation of two well-defined groups: Cluster 1 brings together the closest variables $\left(\mathrm{O}_{3}\right.$, Heat, Evaporation, Minimum Temperature and Maximum Temperature), cluster 2 , the nearest variables $\left(\mathrm{SO}_{2}, \mathrm{CO}, \mathrm{PM} 10\right)$. Time series prediction models were adjusted for the variable $\mathrm{O}_{3}$, using the other variables of group 1 as exogenous. The Temporal Series model that allows the inclusion of exogenous variables (SARIMAX), obtained better prediction results in relation to its competitors (SARIMA and Exponential Smoothing class). It is concluded that the combined use of multivariate analysis models and time series provides better prediction results. It is worth highlighting the wide possibility of using the joint techniques of multivariate analysis and time series, and can be used as a support tool in decision making.

KEYWORDS: Air pollution; Climatic variables; Time series models; Multivariate analysis models.

\section{LA RELACIÓN ENTRE LA CONTAMINACIÓN DEL AIRE Y LAS PRINCIPALES VARIABLES CLIMATOLÓGICAS EN CANOAS-RS}

RESUMEN: Las últimas décadas, junto con el creciente aumento del número de vehículos en Brasil, también ha crecido la preocupación con factores ligados a la contaminación y sus consecuencias para la sociedad. En este contexto, el presente trabajo tuvo por objetivo aplicar técnicas estadísticas de análisis multivariado a los datos de contaminantes del aire y variables meteorológicas para investigar la relación entre estos componentes. Además, se ajustaron modelos de Previsión de series temporales para los índices de contaminación del aire, utilizando las variables climatológicas como exógenas a los modelos, con la finalidad de comparar el desempeño de los modelos. Los datos fueron recolectados junto al Instituto Nacional de Meteorología (INMET) (variables climatológicas) y la Fundación Estadual de Protección Ambiental (FEPAM) (contaminantes del Aire) referente al municipio de Canoas-RS, durante el año 2014. Los resultados del análisis de los resultados El cluster 1 reúne las variables más cercanas $\left(\mathrm{O}_{3}\right.$, Insolación, Evaporación, Temperatura Mínima y Temperatura Máxima), el cluster 2, las variables más cercanas $\left(\mathrm{SO}_{2}, \mathrm{CO}, \mathrm{PM} 10\right)$. Se ajustaron modelos de previsión de series temporales para la variable $\mathrm{O}_{3}$, usando las demás variables del grupo 1 como exógenas. El modelo de Serie Temporal que permite la inclusión de variables exógenas (SARIMAX), obtuvo mejores resultados de previsión en relación a sus competidores (SARIMA y de la clase de Alisamiento Exponencial). Se concluye que el uso conjunto de modelos de análisis multivariada y series temporales proporciona mejores resultados de previsión. Se destaca la amplia posibilidad de utilización de las técnicas conjuntas de análisis multivariada y series temporales, pudiendo ser utilizadas como herramienta de apoyo en las tomas de decisión.

PALABRAS CLAVE: Contaminación del aire. Variables climatológicas. Modelos de series temporales. Modelos de análisis multivariado.

\section{INTRODUÇÃO}

Os poluentes do ar vêm crescendo de forma desordenada e causando inúmeras preocupações a população. Nas cidades de grande e médio porte, estes poluentes se associam as massas de ar e, dessa forma, deslocam-se facilmente, atingindo inúmeras pessoas em períodos curtos de tempo, de acordo com Gioda e Gioda (2006), Barcellos (2009) e Leite et al. (2011).

A degradação do ar decorre de um conjunto de fatores como: as taxas de emissões dos poluentes, a localização, a concentração das fontes emissoras, as características físico-químicas dos poluentes, bem como a dispersão destes na atmosfera e a reação química entre eles, ações que são fortemente influenciadas por condições climatológicas (INSTITUTO DE ENERGIA E MEIO AMBIENTE, 2014). 
Em parte, essa grande concentração de poluentes se deve ao deslocamento de veículos, indústrias e demais setores que por estarem concentrados em uma determinada região acabam contribuindo para os altos índices de poluição. De acordo com Monte, Albuquerque e Reisen (2015), com a industrialização, aliada ao crescimento populacional, especialmente o urbano, vêm aumentando as preocupações relacionadas ao meio ambiente.

De acordo com Moreira, Tirabassi e Moraes (2008), as condições meteorológicas desempenham um papel importantíssimo no transporte e na difusão dos poluentes. Ainda, segundo os autores, modelos matemáticos constituem uma ferramenta importante para auxiliar nas medidas de concentrações de poluentes e para conhecimento de sua evolução. A introdução da modelagem matemática produz um salto de qualidade na gestão da poluição atmosférica em respeito àquela possível somente através de medidas.

Dessa forma, é de suma importância a análise entre as variáveis climatológicas e os principais poluentes do ar, verificando as relações de causa e efeito entre eles, por meio do emprego de técnicas de análise multivariada. Os modelos de séries temporais podem auxiliar no entendimento do comportamento da série de poluentes do ar e realizar previsões futuras a fim de colaborar no planejamento de áreas de fundamental importância como: a saúde, a meteorologia e o desenvolvimento urbano. Portanto, esta pesquisa tem por objetivo aplicar conjuntamente técnicas estatísticas de análise multivariada e previsão de séries temporais aos dados de poluentes do ar e variáveis meteorológicas.

\section{MATERIAIS E MÉTODOS}

\subsection{ANÁLISE DE AGRUPAMENTOS}

A técnica de análise de agrupamentos permite verificar a relação de interdependência entre as variáveis, ou seja, um cluster visa agrupar variáveis com características comuns de maior homogeneidade nos grupos e grande heterogeneidade entre os grupos, sem perder informações de todo o conjunto de dados em estudo Souza e Silva (2009).

Para Vicini (2005) e Saad (2009), a técnica não faz distinção entre variáveis dependentes e independentes, variáveis do tipo causa e efeito. Deste modo, a análise de agrupamentos (cluster analysis) não pressupõe um número nem uma estrutura de conglomerados; toma como base similaridades (ou proximidades) entre itens (objetos, indivíduos ou variáveis), que podem ser, de alguma forma, medidas. Como resultado, obtém-se certo número de conglomerados no qual se agrupam, devido a sua proximidade, de acordo com Rinaldi, Morabito e Tachibana (2009).

Em resumo, a técnica de análise de agrupamento foi utiliza neste trabalho para definir as varáveis climatológicas que mais se aproximam das variáveis de poluentes do ar, ou seja, possuem maior influência, com a finalidade de utilizar as mesmas como variáveis exógenas explicativas nos modelos de previsão de séries temporais que permitam essa inclusão, na busca de melhores resultados de previsões. 


\subsection{ANÁLISE FATORIAL}

A análise fatorial (AF) busca a redução do número de agrupamentos com pouca perda de representatividade das variáveis. Os autovalores de cada eixo representam o quanto de variância original é explicado pelos seus fatores (RINALDI, MORABITO e TACHIBANA ;2009).

A análise fatorial é um conjunto de técnicas que visam tornar os dados observados mais claros, os fatores explicam a variância das variáveis observadas que se revelam pelas correlações entre as variáveis observadas. Em resumo, a AF é um método para determinar o número de fatores existente em um conjunto de dados e serve para determinar quais testes, ou variáveis, pertencem a quais fatores, de acordo com Vicini (2005). O propósito principal da AF é interpretar a estrutura de um conjunto de dados multivariados, a partir da matriz de variância covariância ou da matriz de correlação, segundo Lírio e Souza (2008).

A análise fatorial será utilizada neste trabalho como uma contraprova para Análise de Agrupamento, confirmando resultados propostos nos Cluster e as variáveis a serem utilizadas como variáveis explicativas nos modelos de previsão de séries temporais.

\subsection{MODELOS DE PREVISÃO DE SÉRIES TEMPORAIS}

A análise de séries temporais aplicadas a dados de poluição do ar, associados a dados climatológicos, pode trazer um ganho considerável às pesquisas, já que o clima e a poluição interferem diretamente na qualidade de vida dos habitantes de qualquer região, e é uma preocupação constante e crescente.

De acordo com Batista (2009), no estudo de uma série histórica, buscase investigar o mecanismo gerador da série temporal, fazer previsões de valores futuros, descrever o seu comportamento, avaliar os fatores que influenciam no seu comportamento, buscando relações de causa e efeito, além de construir um modelo apropriado no intuito de se realizar previsões para embasar tomadas de decisão.

Para Morettin e Toloi (2004), a previsão não constitui um fim em si, mas um meio de fornecer informações e subsídios para uma consequente tomada de decisão, visando atingir determinados objetivos e reduzir as incertezas no processo de tomadas de decisões.

Com auxílio de modelos de séries temporais, ajustou-se modelos para realizar previsões futuras dos principais índices de poluição do ar (modelos univariados) e no modelo multivariado utilizou-se as variáveis climatológicas, definidas pela análise de agrupamento e fatorial, como variáveis exógenas ao modelo, com intuito de melhorar a qualidade das previsões.

Os modelos testados e comparados as variáveis em estudo foram os modelos da classe de alisamento exponencial (Simples, Bi-Paramétrico e de Holt-Winters). Da classe Box Jenkins (Autorregressivos Integrados e de Médias Móveis (ARIMA/SARIMA) e o modelo autorregressivo com médias móveis e entrada de variáveis exógenas (ARMAX), descritos em Reisen, Molinares e Teixeira (2008), Morettin e Toloi (2004), Morettin e Toloi (1987), Martínez e 
Zamprogno (2003), Silva (2011) e Ozaki e Admi (2012), Chechi e Sanches (2013), Lizzi (2012), Lúcio et al. (2010), Nascimento (2013) e Veríssimo et al. (2012).

\subsection{CRITÉRIOS DE SELEÇÃO DOS MODELOS BOX JENKINS}

Os critérios utilizados para verificar o ajuste dos modelos ARIMA/SARIMA e ARMAX são: AIC proposto por Akaike (1974) e BIC desenvolvido por Akaike (1978), relatados abaixo.

Tabela 1 - Critérios de avaliação da qualidade do ajuste

\begin{tabular}{cc}
\hline Critério & Equação \\
\hline AIC (Akaike Information Criteria) & $\mathrm{AIC}=\ell \mathrm{n}\left(\hat{\sigma}_{\mathrm{e}}^{2}\right)+\frac{2(\mathrm{p}+\mathrm{q})}{\mathrm{n}}$ \\
$\mathrm{BIC}$ (Bayesian Information Criterion) & $\mathrm{BIC}=\ell \mathrm{n}\left(\hat{\sigma}_{\mathrm{e}}^{2}\right)+\frac{(\mathrm{p}+\mathrm{q}) \ell \mathrm{n}(\mathrm{n})}{\mathrm{n}}$, \\
\hline
\end{tabular}

Fonte: Adptado de Akaike (1974 e 1978).

sendo $\hat{\sigma}_{e}^{2}$ a variância estimada dos erros, n o tamanho da amostra e $(p, q)$ o número de parâmetros de cada componente AR e MA. Os critérios levam em conta a variância do erro, o tamanho da amostra $n$ e os valores de $(p, q)$. Quanto menores forem esses valores, melhor é a adequação do modelo para realizar previsões.

Além disso, a condição de ruído branco dos resíduos é considerada, ou seja, faz-se necessário que os erros do modelo tenham média zero e variância constante, além de serem descorrelacionados ao longo do tempo.

\subsection{CRITÉRIOS DE SELEÇÃO DOS MODELOS DA CLASSE DE ALISAMENTO EXPONENCIAL E COMPARAÇÃO ENTRE CLASSES DE MODELOS}

Os critérios de avaliação da qualidade das previsões a serem utilizados para os modelos de séries temporais descritos acima são: Erro Quadrático Médio (EQM), Soma dos Quadrados dos Erros (SSE), Média Absoluta dos Erros (MAD) e o Erro Absoluto Médio Percentual (MAPE), descritos na Tabela 2, de acordo com Tidre, Biase e Silva (2013), Esquivel, Senna e Gomes (2012), Barbosa e Chaves (2012) e Feliciano (2009).

Tabela 2 - Critérios de avaliação da qualidade de previsão

\section{Critério}

Erro quadrático médio

Soma dos quadrados dos Erros

\section{Equação}

$\mathrm{EQM}=\frac{\sum_{\mathrm{t}=1}^{\mathrm{n}}\left(\mathrm{Z}_{\mathrm{t}}-\hat{\mathrm{Z}}_{\mathrm{t}}\right)^{2}}{\mathrm{n}}$

$\mathrm{SSE}=\sum_{\mathrm{t}=1}^{\mathrm{n}}\left(\mathrm{Z}_{\mathrm{t}}-\hat{\mathrm{Z}}_{\mathrm{t}}\right)^{2}$ 
Média absoluta dos erros

Erro Absoluto Médio Percentual

$$
\begin{array}{r}
\text { MAD }=\frac{\sum_{\mathrm{t}=1}^{\mathrm{n}}\left|\mathrm{Z}_{\mathrm{t}}-\hat{\mathrm{Z}}_{\mathrm{t}}\right|}{\mathrm{n}} \\
\mathrm{MAPE}=\frac{\sum_{\mathrm{t}=1}^{\mathrm{n}}\left|\frac{\mathrm{Z}_{\mathrm{t}}-\hat{\mathrm{Z}}_{\mathrm{t}} \mid}{Z_{t}}\right|}{\mathrm{n}} .100 \%
\end{array}
$$

sendo $Z_{t}$ o valor da série no instante $t ; \hat{Z}_{t}$ o valor previsto para o instante $t ; n$ o número período de tempos.

Quanto menores os valores encontrados nos critérios (EQM), (SSE), (MAD) e o (MAPE), mais próximos dos valores reais estão os valores previstos pelos modelos ajustados. Os critérios descritos na tabela 2, vão determinar 0 melhor modelo entre os testados para representar a série de poluente do ar e realizar previsões de poluentes do ar.

\subsection{DESCRIÇÃO DOS DADOS}

Para realização deste estudo, foram considerados os dados das séries históricas, fornecidos pela Fundação Estadual de Proteção Ambiental - RS FEPAM, das medições horárias dos seguintes poluentes do ar: material particulado (PM10), ozônio $\left(\mathrm{O}_{3}\right)$, dióxido de enxofre $\left(\mathrm{SO}_{2}\right)$ e monóxido de carbono (CO), realizadas no município de Canoas-RS, no ano de 2014. As variáveis meteorológicas são: Umidade Relativa Média do Ar (\%), Temperatura Mínima $\left({ }^{\circ} \mathrm{C}\right)$, Temperatura Máxima $\left({ }^{\circ} \mathrm{C}\right)$, Temperatura Média $\left({ }^{\circ} \mathrm{C}\right)$, Precipitação $(\mathrm{mm})$, Insolação $(\mathrm{h})$, Evaporação $(\mathrm{mm})$ e Velocidade do Vento $(\mathrm{m} / \mathrm{s})$ coletados diariamente pelo Instituto Nacional de Meteorologia (INMET). Os dados disponíveis foram transformados em médias diárias.

Cabe ressaltar que outras séries históricas de poluentes do ar dos municípios de Porto Alegre-RS, Esteio-RS e próprio Canoas-RS, (para diferentes períodos) foram analisadas. Entretanto o grande número de dados faltantes impossibilitou o uso das mesmas.

Para realizar a comparação dos modelos de série temporal mais apropriado para previsão, foram reservadas as 20 últimas observações das séries históricas em estudo, ou seja, dias não contabilizados no ajuste dos modelos e usados os critérios: MAPE, EQM, MAD e SSE, descritos na tabela 2. Como software de apoio foram utilizados o STATISTICA 9.1 e o R.

\section{RESULTADOS E DISCUSSÃO}

Inicialmente são apresentadas as medidas descritivas dos poluentes do ar. Os valores das médias e desvios padrões das variáveis, coeficiente de variação e valor máximo e mínimo, utilizados neste estudo, estão expostos na Tabela 3. Observa-se que há um alto coeficiente de variação, principalmente nas variáveis $\mathrm{CO}$ e $\mathrm{SO}_{2}$, indicando que os dados em estudo são heterogêneos, ou seja, demonstram um comportamento com variabilidade considerável durante o ano, o que remete a necessidade de estudos dessas variáveis para entender os 
seus comportamentos e se essa variabilidade está associada a fatores externos (variáveis climáticas). A variável $\mathrm{O}_{3}$ indica um comportamento mais próximo a homogêneo, ou seja, uma menor variabilidade durante o período de estudo, indicando que os dados se aproximam em maior número da média em relação as outras poluentes do ar.

Tabela 3 - Análise descritivas dos poluentes do ar

\begin{tabular}{cccccc}
\hline Variáveis & Média & Desvio-Padrão & $\begin{array}{c}\text { Coeficiente de } \\
\text { variação }\end{array}$ & Mínimo & Máximo \\
\hline $\mathrm{CO}\left(\mu \mathrm{g} / \mathrm{m}^{3}\right)$ & 0,06 & 0,063 & 105 & 0,03 & 0,42 \\
$\mathrm{O}_{3}\left(\mu \mathrm{g} / \mathrm{m}^{3}\right)$ & 25,77 & 10,22 & 39,6 & 2,3 & 55,86 \\
$\mathrm{PM}_{10}\left(\mu \mathrm{g} / \mathrm{m}^{3}\right)$ & 31,43 & 17,62 & 56,06 & 4,34 & 133,37 \\
$\mathrm{SO}_{2}\left(\mu \mathrm{g} / \mathrm{m}^{3}\right.$ & 1,33 & 1,73 & 130 & 0,65 & 21,05 \\
\hline
\end{tabular}

Dando início a análise de agrupamento, utilizou-se como processo de aglomeração hierárquica o método de ligação completo (na busca de maior homogeneidade dentro de cada grupo), sendo a métrica de similaridade a Correlação Person ( $r$.

No dendograma é apresentado o comportamento de todas as variáveis consideradas no estudo e a formação de dois grupos, os quais possuem as variáveis de maior relevância dentro do conjunto em estudo (Figura 1A). O ponto de corte para a formação dos grupos foi definido pelo método gráfico, considerando o ponto de inflexão da curva quando esta passa a ser praticamente paralela ao eixo das abscissas, excluindo as componentes que restam, pois possuem pouca informação (Figura 1B).

A

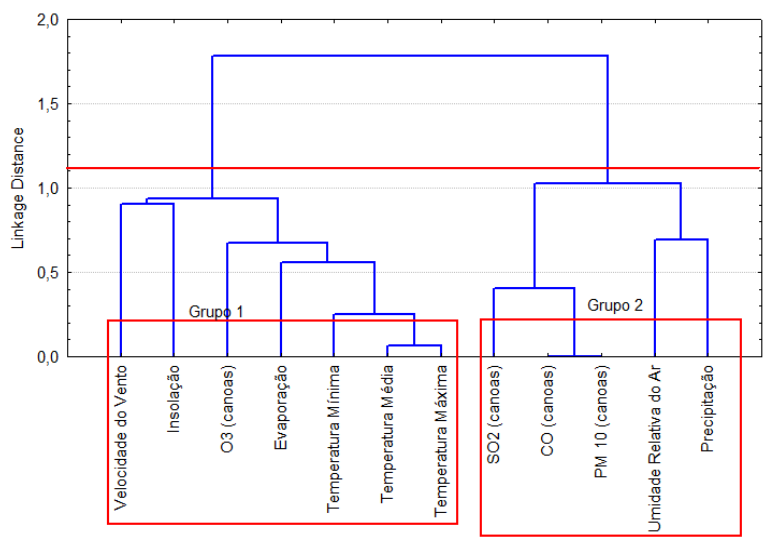

B

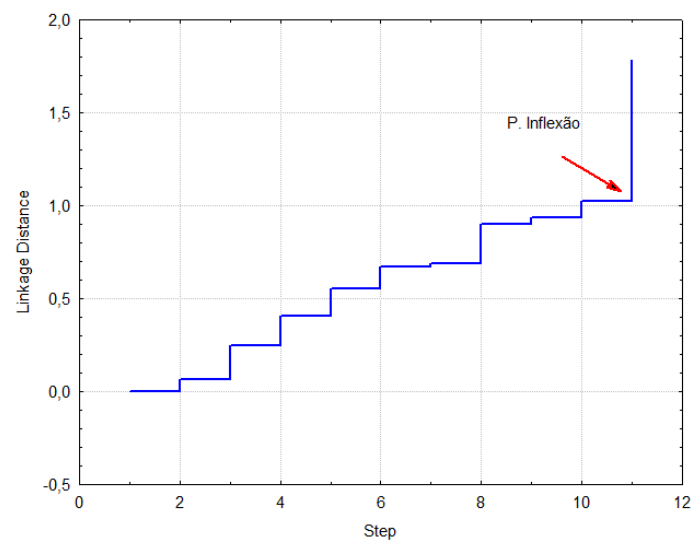

Figura 1 - Dendograma (1A) e Scree plot (1B).

Realizando-se um corte na altura 1,2 de acordo com o método gráfico (Figura 1B), obtém-se o grupo 1 com 7 variáveis e o grupo 2 com 5 variáveis.

O cluster 1 , representado pelo grupo 1 , reúne as variáveis mais próximas: Velocidade do Vento, Insolação, $\mathrm{O}_{3}$, Evaporação, Temperatura Média e Temperatura Máxima e Mínima. O cluster 2, representado pelo grupo 2, reúne as variáveis mais próximas: $\mathrm{SO}_{2}, \mathrm{CO}, \mathrm{PM} 10$, Umidade Relativa do $\mathrm{Ar} \mathrm{e}$ Precipitação.

Para a composição de cada grupo, verifica-se a importância de cada variável por meio da $A F$. Os resultados mostram a relação dos fatores com as 
variáveis originais (Figura 2), onde é apresentado no plano fatorial a relação das componentes principais com as variáveis originais e de que forma essas variáveis se agrupam, confirmando os resultados dos agrupamentos formados e apresentados no dendograma da Figura 1A. Por exemplo, verifica-se baixa correlação entre Velocidade do Vento (W) no Grupo 1 e UR e Precipitação com o Grupo 2. Cargas fatoriais próximas para temperatura máxima, média e mínima, no grupo 1 e no grupo 2 há uma proximidade entre PM10 e CO, como pode ser observado na figura 2.

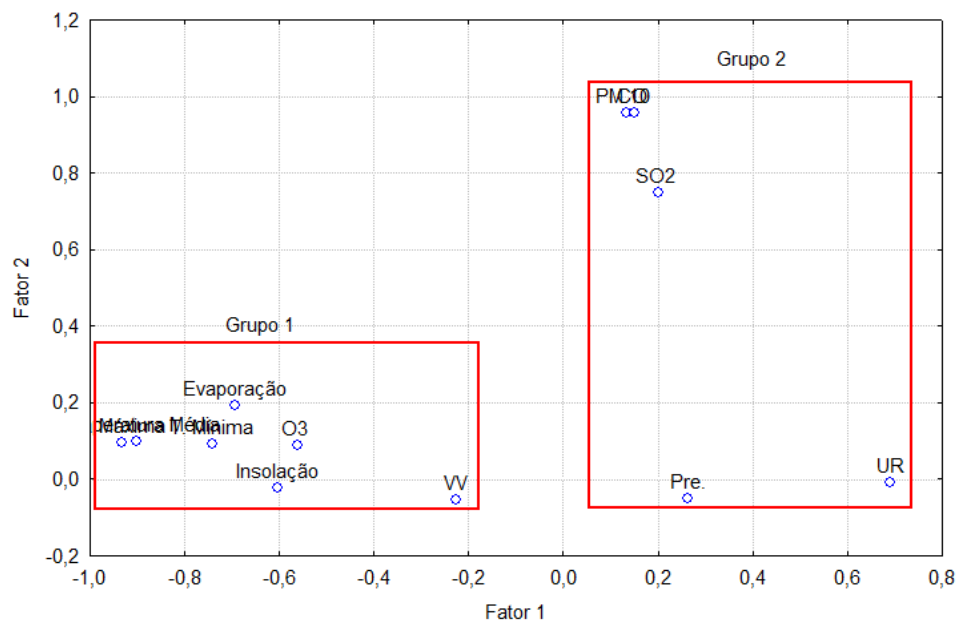

Figura 2 - Plano Fatorial - Fator 1 vs Fator 2.

Como o intuito é utilizar as variáveis climatológicas para auxiliar nas previsões das varáveis de poluentes do ar, é necessário encontrar o máximo de homogeneidade em seus grupos. Dessa forma, foram excluídas as variáveis Precipitação e UR no grupo 2 e Velocidade do Vento no grupo 1, tendo em vista possuírem baixa correlação (homogeneidade dentro dos seus grupos), a variável Temperatura Média será excluída pela proximidade com a Temperatura Máxima e Mínima, possuindo a mesma carga de informação.

Na Figura 3 demonstra-se a nova formação dos grupos no dendograma (Figura 3A) e o Scree plot (Figura 3B), que define o ponto de corte e confirma as componentes dos grupos 1 e 2 .

A

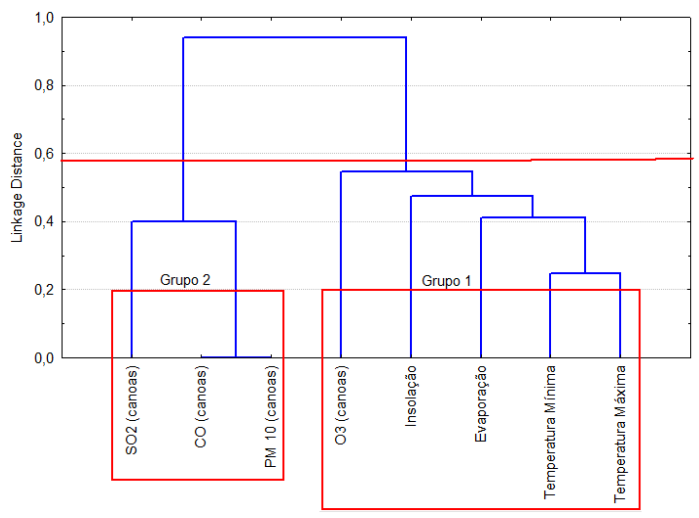

B

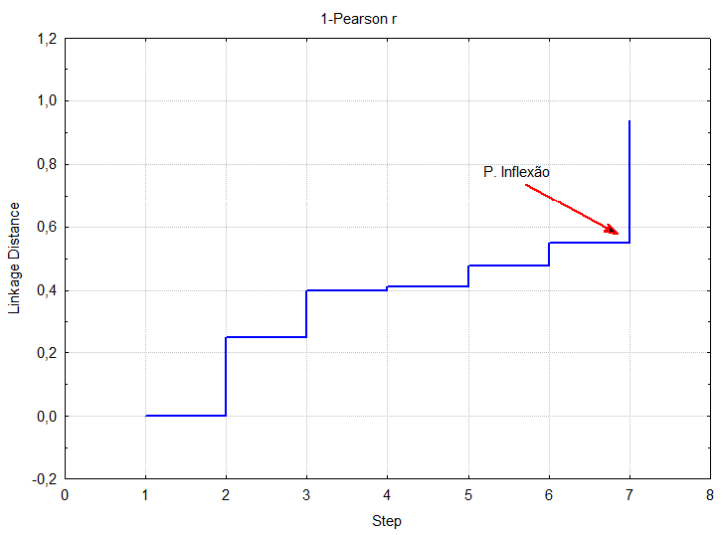

Figura 3 - Dendograma (3A) e Scree plot (3B). 
Realizando-se um corte na altura 0,6 de acordo com o método gráfico, obtêm-se os grupos finais definidos.

O cluster 1 , representado pelo grupo 1 , reúne as variáveis mais próximas: $\mathrm{O}_{3}$, Insolação, Evaporação, Temperatura Mínima e Temperatura Máxima. O cluster 2, representado pelo grupo 2, não demostrou influências das variáveis climatológicas e reúne as variáveis mais próximas: $\mathrm{SO}_{2}, \mathrm{CO}, \mathrm{PM} 10$. $\mathrm{O}$ Plano Fatorial (Figura 4), também corrobora e confirma a formação dos grupos.

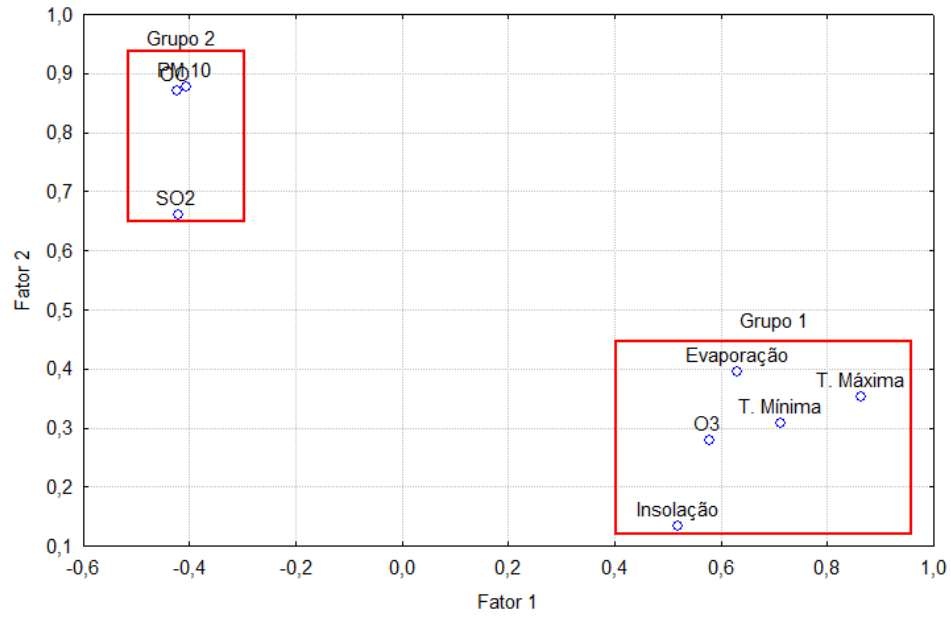

Figura 4 - Plano Fatorial - Fator 1 vs Fator 2.

Com os grupos definidos, o próximo passo foi definir as variáveis de poluição do ar, que serão modeladas para a previsão, e as variáveis utilizadas como exógenas aos modelos.

Devido às características de agrupamentos e por ser um dos principais poluentes do ar, no grupo 1 , serão construídos modelos de previsão para a variável $\mathrm{O}_{3}$ - Ozônio e como variáveis explicativas: Insolação, Evaporação, Temperatura Mínima e Temperatura Máxima que devido à proximidade constatada com $\mathrm{O}_{3}$ podem contribuir no ajuste dos modelos de previsão e consequentemente obter melhores resultados de previsão dos modelos Autorregressivos Integrados e de Médias Móveis que permitem a entrada de variáveis exógenas/explicativas na forma de uma estrutura de regressão.

Observa-se que as variáveis meteorológicas apresentam relação linear com as concentrações de $\mathrm{O}_{3}$. Os índices de $\mathrm{O}_{3}$ estão associados às mudanças dessas variáveis, ou seja, o aumento da Insolação, Temperatura mínima e máxima acarreta aumento nas concentrações de $\mathrm{O}_{3}$ e 0 aumento da UR e precipitação acarreta diminuição das concentrações de $\mathrm{O}_{3}$. Essas relações também são discutidas em Santos et al. (2017) e Santos, Reboita e Carvalho (2018), através da análise componentes principais.

No grupo 2, como as variáveis $\mathrm{SO}_{2}, \mathrm{CO}, \mathrm{PM} 10$ não são influenciadas diretamente por variáveis climáticas, neste trabalho não foram construídos modelos de Previsão de Séries Temporais para o grupo 2.

Com os grupos definidos pela análise multivariada de cluster e fatorial, o próximo passo foi ajustar os modelos de séries temporais para a variável $\mathrm{O}_{3}$. Todos os modelos de previsão foram ajustados para uma previsão de 20 passos à frente, ou seja, dias que não foram contabilizados no ajuste dos modelos. 


\subsection{AJUSTE DOS MODELOS DE SÉRIE TEMPORAL SARIMA/SARIMAX}

Para dar-se início ao ajuste do modelo, observa-se, na Figura 5, o comportamento da série original, buscando identificar suas componentes, como tendência e/ou sazonalidade.

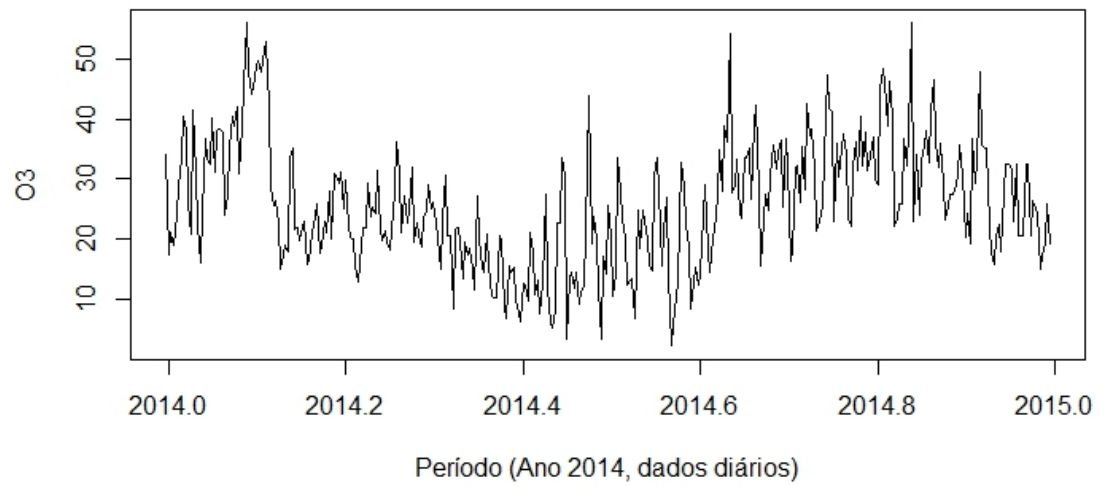

Figura 5 - Gráfico da série observada $\mathrm{O}_{3}$.

Analisando a Figura 5, verifica-se que a série possui indícios de sazonalidade e não estacionariedade, sendo necessário aplicar diferenciações na série original.

Após a etapa inicial de análise gráfica dos dados, foram realizados testes estatísticos para confirmar as hipóteses levantadas na análise gráfica. De acordo com o teste de sequências $p$-valor $<0,05$, a série apresenta indícios de não ser estacionária. Para avaliar a sazonalidade, foi realizado o teste Kruskal-Wallis e de Friedman, sendo rejeitada a hipótese nula $\mathrm{H}_{-} \mathrm{O}$ ( $\mathrm{p}$-valor $<0,001$ ) e aceita-se a hipótese alternativa $H_{\_} 1$ de presença de efeito sazonal na série.

Ao término da etapa de análise inicial da série $\mathrm{O}_{3}$, identificou-se a presença de sazonalidade e de não estacionariedade na série. A próxima etapa foi a construção dos gráficos de Funções de autocorrelação (FAC) e de autocorrelação parcial (FACP).

Os gráficos das FAC e de FACP, demonstram as correlações seriais no domínio do tempo. Com base em suas frequências estimadas, é possível verificar sazonalidades e ciclos na série. A Figura 6, apresenta os correlogramas da série, sem diferença e com diferença de ordem 1. 


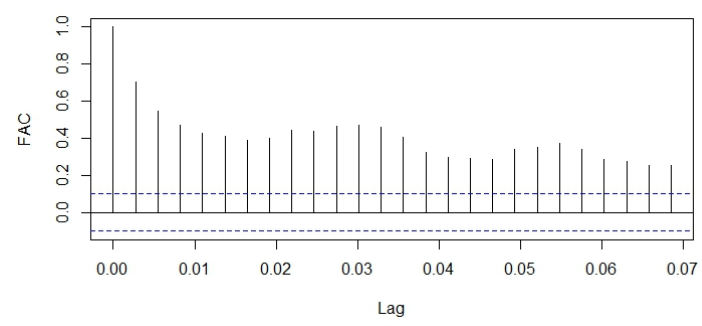

Série Diferenciada $(\mathrm{d}=1)$

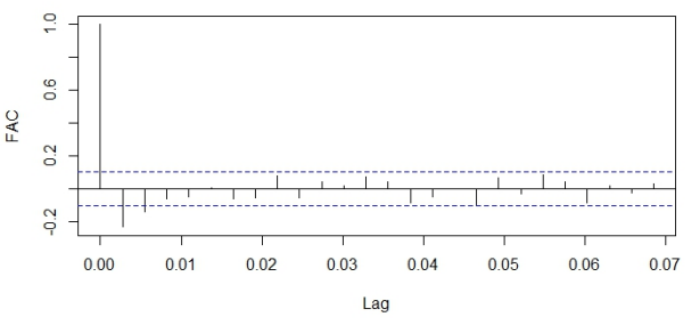

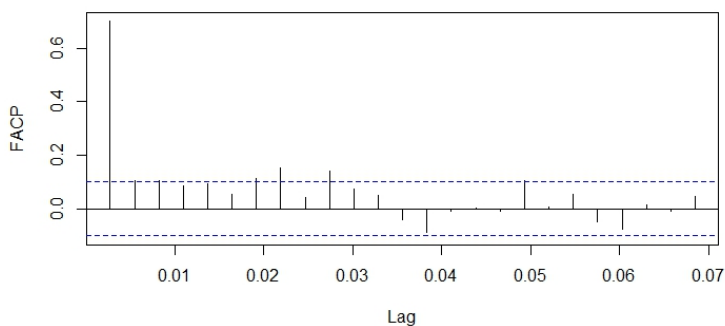

Série Diferenciada $(\mathrm{d}=1)$

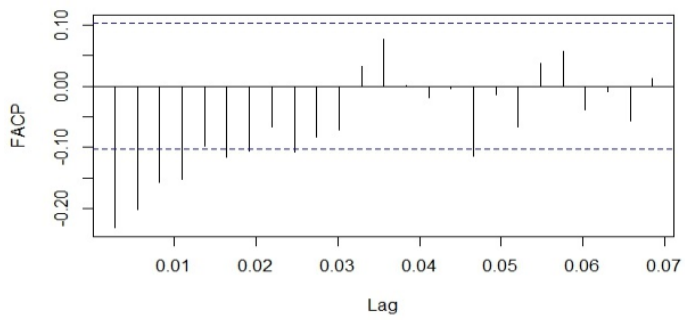

Figura 6 - Função de Autocorrelação e Autocorrelação Parcial.

A FAC em nível corrobora com os testes estatísticos que a série não é estacionária, demostrando fortes correlações entre os lag, sendo necessário aplicar diferenciações para estabilizar a série, nota-se também que existe sazonalidade de período 12. Ao se analisar os FAC e FACP com diferença de ordem 1, percebem-se características de um modelo estacionário autorregressivo de ordem 1 a 3 e de médias móveis de ordem 1 ou 2 . Os testes estatísticos e a análise gráfica indicam que um modelo do tipo SARIMA/SARIMAX deve ser considerado. Essa análise é sumária e serve para dar início ao processo de modelagem, embora vários modelos de ordens diferentes foram considerados e testados.

$\mathrm{Na}$ Tabela 4, estão expostos modelos concorrentes SARIMA ajustados para a série $\mathrm{O}_{3}$, com os respectivos valores de AIC e BIC, e os parâmetros do modelo.

Tabela 4 - Modelos concorrentes SARIMA e os respectivos critérios para a série $\mathrm{O}_{3}$

\begin{tabular}{cccc}
\hline SARIMA & Parâmetros & \multicolumn{2}{c}{ Critérios de seleção } \\
\cline { 3 - 4 }$(p, d, q)(P, D, Q)$ & & AIC & BIC \\
\hline & $\phi_{1}=0,4849$ & & \\
$(1,1,1)(0,1,1)$ & $\emptyset_{1}=-0,9115$ & 2298,33 & 2313,55 \\
& $\Theta_{1}=-0,9332$ & &
\end{tabular}




$$
\begin{aligned}
\phi_{1} & =0,4723 \\
\phi_{2} & =-0,0333 \\
\phi_{1} & =-0,9147 \\
& \Phi_{1}=-0,053 \\
\Phi_{2} & =-0,0376 \\
\Theta_{1} & =0,1621
\end{aligned}
$$

Considerando os dois melhores modelos significativos ajustados, analisou-se a condição de ruído branco (média zero, variância constante, erros não correlacionados). Na análise dos resíduos o modelo SARIMA $(1,1,1)(0,1,1)$, atende aos pressupostos exigidos.

Os testes de autocorrelação dos resíduos Ljung-Box ( $p$-valor $=0,9949$ ) e Box-Pierce ( $p$-valor $=0,9949)$, do melhor modelo SARIMA $(1,1,1)(0,1,1)$, de acordo com os critérios de seleção AIC e BIC, indicam que os erros do modelo ajustado são descorrelacionados ao longo do tempo.

Analisando a Figura 7, gráfico de FAC e FACP, pode se perceber que não há autocorrelação significativamente diferente de zero em nenhuma defasagem, portanto, os dados são independentes e demonstram um bom ajuste do modelo, confirmando os resultados dos testes de Ljung-Box e Box-Pierce.
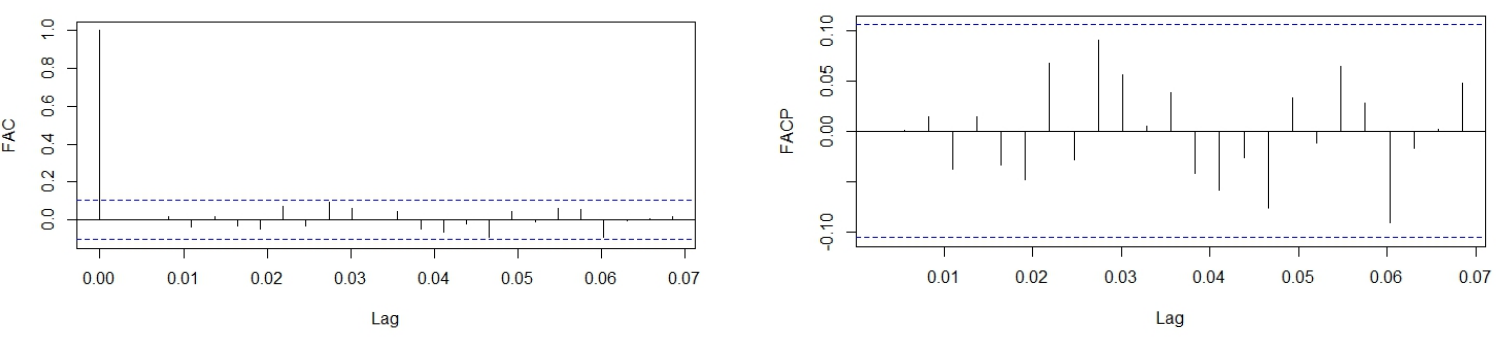

Figura 7 - Função de autocorrelação e autocorrelação parcial dos resíduos.

Os testes de normalidade dos resíduos Jarque-Bera ( $p$-valor $=0,0977$ ) e Shapiro-Wilk ( $p$-valor $=0,1139$ ) demonstram que os resíduos do modelo SARIMA $(1,1,1)(0,1,1)$ seguem distribuição normal.

Considerando a análise residual e os critérios AIC e BIC, o modelo SARIMA $(1,1,1)(0,1,1)$, com um parâmetro autorregressivo $(p)$ explicado pelas observações de um dia passado do $\mathrm{O}_{3}$, um parâmetro de diferenças $(d)$ a fim de tornar a série estacionária na parte ordinal, um parâmetro de médias móveis $(q)$ explicado pelos erros das previsões passadas na parte ordinal, um parâmetro de diferenças (D) na parte sazonal tendo em vista a variância não ser constante ao longo do tempo na parte sazonal e um parâmetro de médias móveis $(Q)$ na parte sazonal, atende a todas as condições de ruído branco e normalidade dos resíduos e concluem a última etapa da escolha do modelo para realizar previsões futuras.

Nessa etapa do trabalho, foram obtidas as previsões da série $\mathrm{O}_{3}$ para um horizonte de 20 passos à frente, ou seja, dias que não foram considerados no ajuste do modelo. Na Figura 8, é demonstrada a série real, ajustada, e os 
valores previstos para os limites de previsão com intervalo de confiança de $95 \%$. As comparações do desempenho do modelo, de acordo com os critérios descritos em metodologia, estão expostas na tabela 6.

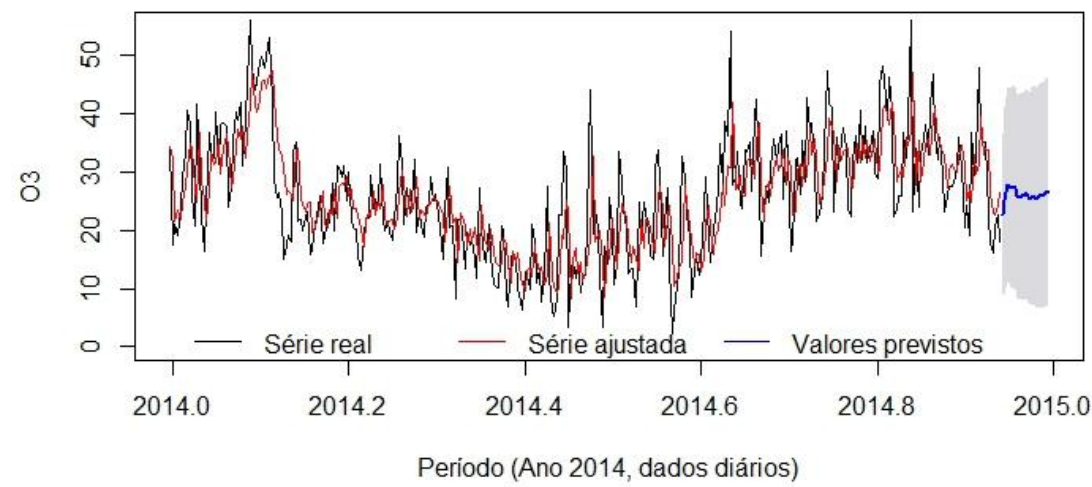

Figura 8 - Modelo SARIMA $(1,1,1)(0,1,1)$ ajustado à série observada.

Para o ajuste do modelo SARIMAX, foi utilizada a variável endógena $\mathrm{O}_{3}$, e foram utilizadas as variáveis exógenas Insolação, Evaporação, Temperatura Mínima e Temperatura Máxima, definidas pela análise multivariada de agrupamento e fatorial, com o objetivo de melhorar as previsões da variável em estudo.

Dentre os diversos modelos testados, na Tabela 5, estão apresentados os dois modelos com melhor desempenho nos critérios de seleção AIC e BIC.

Tabela 5 - Modelos concorrentes SARIMAX e os respectivos critérios (variável $\mathrm{O}_{3}$ )

\begin{tabular}{|c|c|c|c|}
\hline \multirow{2}{*}{$\begin{array}{c}\text { SARIMAX } \\
(p, d, q, r[])(P, D, Q)\end{array}$} & \multirow{2}{*}{ Parâmetros } & \multicolumn{2}{|c|}{ Critérios de seleção } \\
\hline & & AIC & $\mathrm{BIC}$ \\
\hline \multirow{6}{*}{$(1,1,1[1,1,1]))(0,1,1)$} & $\phi_{1}=0,4634$ & \multirow{6}{*}{2207,67} & \multirow{6}{*}{2234,31} \\
\hline & $\emptyset_{1}=-0,9178$ & & \\
\hline & $\Theta_{1}=-0,9312$ & & \\
\hline & T. $\max .=0,3093$ & & \\
\hline & T. $\min .=0,1263$ & & \\
\hline & Evap. $=-0,1262$ & & \\
\hline \multirow{5}{*}{$(1,1,2[1,1,1]))(0,1,1)$} & $\phi_{1}=0,3873$ & \multirow{5}{*}{2209,50} & \multirow{5}{*}{2239,69} \\
\hline & $\emptyset_{1}=-08267$ & & \\
\hline & $\emptyset_{2}=-0,0759$ & & \\
\hline & $\Theta_{1}=0,9328$ & & \\
\hline & T. $\max .=0,3134$ & & \\
\hline
\end{tabular}


T. $\min .=0,0928$

Evap. $=-0,1277$

Entre as variáveis exógenas encontradas na análise de agrupamento e fatorial, por falta de ajuste na construção dos modelos, descartou-se, nesta etapa a variável Insolação. Considerando os dois melhores modelos significativos ajustados, analisou-se a condição de ruído branco. Na análise dos resíduos do modelo SARIMAX $(1,1,1[1,1,1])(0,1,1)$, observou-se que o modelo atendeu os pressupostos indicados para o modelo.

O sinal positivo dos parâmetros da temperatura máxima e mínima indica que há uma relação direta entre $\mathrm{a}_{3}$ e essas variáveis, ou seja, o aumento da temperatura máxima e mínima indica um aumento da concentração de $\mathrm{O}_{3}$, os resultados encontrados estão de acordo com os debatidos em Monte, Albuquerque e Reisen (2015) e Santos et al. (2017). O coeficiente negativo da evaporação indica uma relação inversa, aumento da evaporação acarreta em diminuição da $\mathrm{O}_{3}$, provavelmente devido ao aumento da umidade relativa do $\mathrm{Ar}$, conforme descrito em Carvalho et al. (2004), a uma relação negative entre $\mathrm{O}_{3} \mathrm{e}$ UR.

Os testes de autocorrelação dos resíduos Ljung-Box ( $p$-valor $=0,7046$ ) e Box-Pierce ( $p$-valor $=0,7079)$, do melhor modelo SARIMAX $(1,1,1[1,1,1])(0,1,1)$, de acordo com os critérios de seleção AIC e BIC, indicam que os erros do modelo ajustado são descorrelacionados ao longo do tempo.

Analisando a Figura 9, gráfico de FAC e FACP, percebe-se que não há autocorrelação significativamente diferente de zero em nenhuma defasagem, portanto, os dados são independentes e demonstram um bom ajuste do modelo, confirmando os resultados dos testes de Ljung-Box e Box-Pierce.
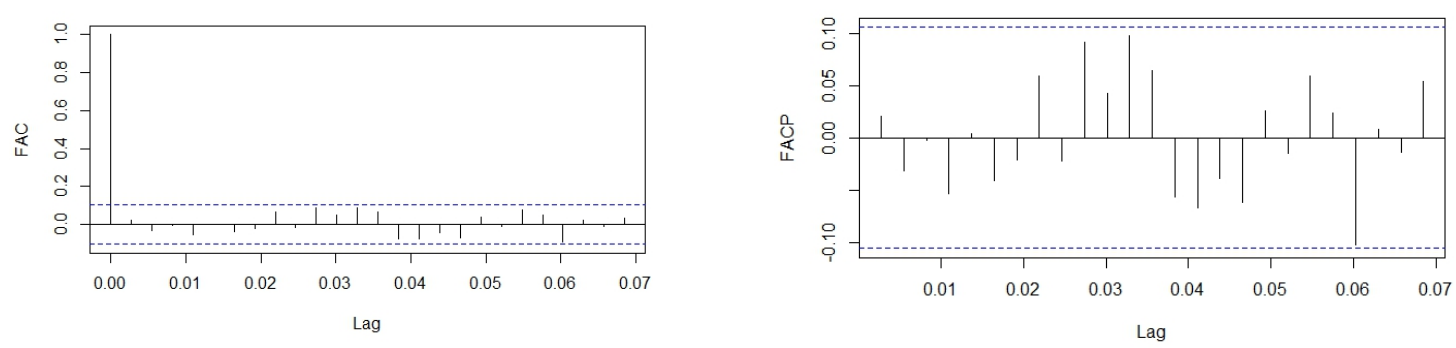

Figura 9 - Função de autocorrelação e autocorrelação parcial dos resíduos do modelo SARIMAX.

Os testes de normalidade dos resíduos Jarque-Bera ( $p$-valor $=0,02841$ ) e Shapiro-Wilk ( $p$-valor $=0,1801$ ) demonstram que os resíduos do modelo SARIMAX $(1,1,1[1,1,1])(0,1,1)$ seguem distribuição normal.

Considerando a análise residual e os critérios AIC e BIC, o modelo SARIMAX, com um parâmetro autorregressivo $(p)$, um parâmetro de diferenças (d), um parâmetro de médias móveis (q) na parte ordinal, um parâmetro de diferenças (D), um parâmetro de médias móveis (Q) na parte sazonal e um parâmetro das variáveis exógenas Temperatura Máxima, Temperatura Mínima e Evaporação, o modelo atende a todas as condições de ruído branco e 
normalidade dos resíduos e concluem a última etapa da escolha do modelo para realizar previsões futuras.

Nessa etapa do trabalho, foram obtidas as previsões da série $\mathrm{O}_{3}$ para um horizonte de 20 passos à frente, ou seja, dias que não foram considerados no ajuste do modelo.

Na Figura 10, é demonstrada a série real, ajustada e os valores previstos para os limites de previsão com intervalo de confiança de $95 \%$, pode ser observado o bom ajuste do modelo. A inclusão das variáveis explicativas temperatura máxima/mínima e evaporação, proporciona uma aproximação mais adequada entre a série ajustada e observada e consequentemente resultados de previsão mais próximos ao real. As comparações do desempenho do modelo, de acordo com os critérios descritos em metodologia, estão expostas na tabela 6 .

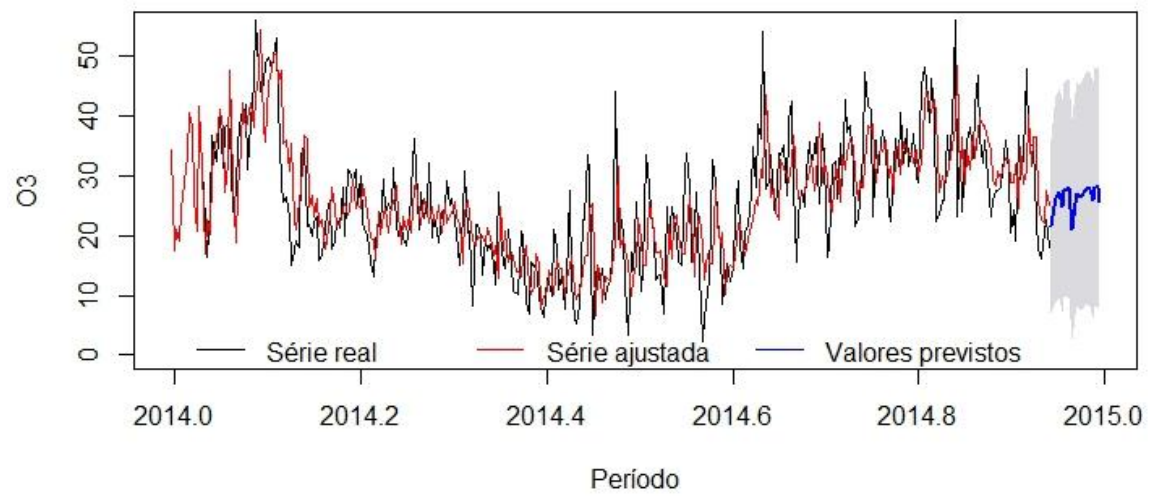

Figura 10 - Modelo SARIMAX $(1,1,1[1,1,1])(0,1,1)$ ajustado à série observada.

\subsection{AJUSTE DOS MODELOS DA CLASSE DE ALISAMENTO EXPONENCIAL}

No ajuste do modelo de alisamento exponencial simples, encontrou-se o parâmetro de suavização alfa $a=0,5249$. A previsão de todos os valores futuros da série é dada pela última constante de alisamento.

Na Figura 11, é demonstrada a série real, ajustada e os valores previstos para os limites de previsão com intervalo de confiança de 95\%. A série representada pela cor verde é a série ajustada, as circunferências de cor preta, representam a série $\mathrm{O}_{3} \mathrm{e}$, em azul, as previsões futuras.

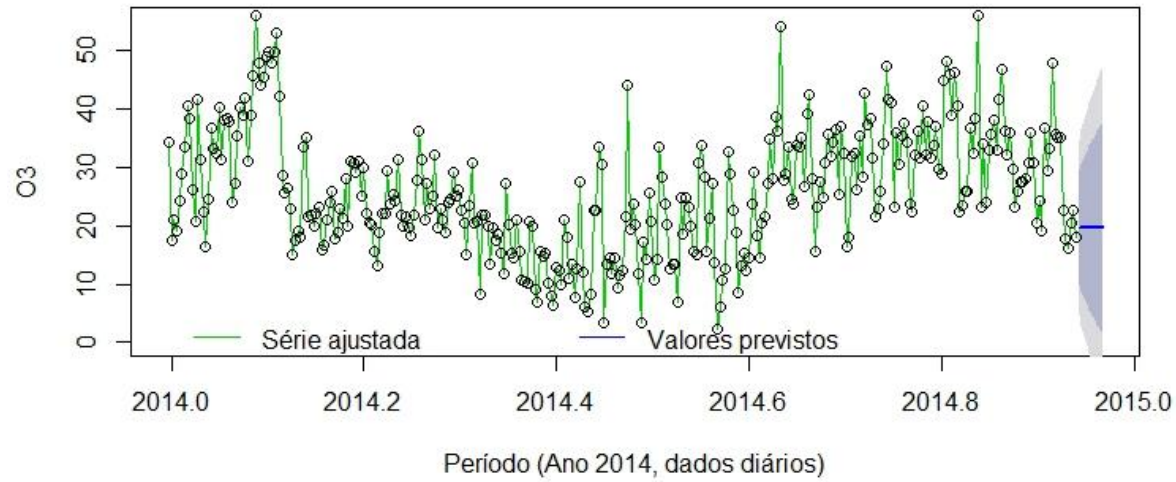

Figura 11 - Modelo de alisamento exponencial simples. Fonte: Os autores. 
O ajuste do modelo de Alisamento exponencial simples é adequando a série em estudo. Entretanto como a previsão é dada pela última constante de alisamento, ou seja, a previsão é linear, os resultados previstos não acompanham a sazonalidade da série e, portanto, acabam se afastando da série observada, não produzido bons resultados de previsão, conforme exposto na tabela 6.

O Alisamento exponencial biparamétrico é uma técnica similar ao alisamento exponencial simples, suavizando diretamente os valores da tendência, permitindo usar constantes com diferentes valores para o nível e tendência. O valor encontrado para a constante em nível é de $A=0,5254$ e, para a tendência beta $C=0,0001$.

Na Figura 12, encontra-se a série ajustada e os valores previstos para os limites de previsão com intervalo de confiança de $95 \%$. A série representada pela cor vermelha é a série ajustada, as circunferências de cor preta, representa a série $\mathrm{O}_{3}$ e, em azul, as previsões futuras.

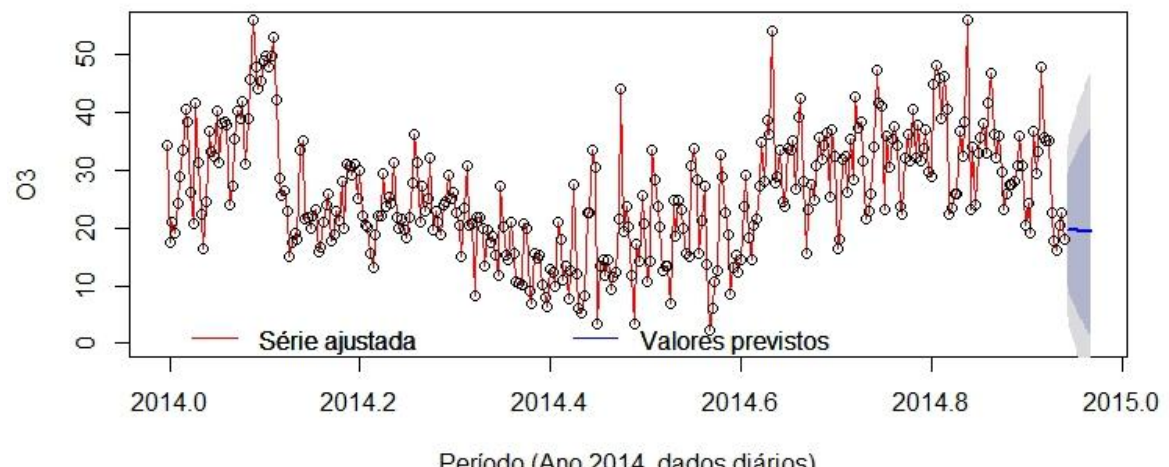

Figura 12 - Modelo de alisamento biparamétrico ajustado a variável $\mathrm{O}_{3}$. Fonte: Os autores.

O modelo de alisamento exponencial biparamétrico permite ajustar uma componente para a tendência. Como verificado nos testes inicias a série em estudo não possui tendência significativa, valor esse confirmado pela componente da tendência do modelo ajustado $(C=0,0001)$. Desta forma a inclusão da tendência no modelo ajustado e nas previsões não acresce em melhores resultados de ajuste e previsão. As comparações estão na tabela 6 .

A técnica de alisamento exponencial de Holt-Winters é indicada para séries que apresentam tendências e/ou sazonalidade. De acordo com as características da série, é ajustado um modelo aditivo (séries homogêneas) ou multiplicativo (séries heterogêneas) e, posteriormente, feita a comparação, utilizando os critérios de seleção.

No ajuste dos modelos, os valores encontrados para as constantes de suavização do modelo aditivo foram: $A=0,6576$ (constante de suavização do nível); $C=0,0000$ (constante de suavização da tendência) e $D=0,8467$ (constante de suavização da sazonalidade) e, para o modelo multiplicativo, foi: $A=0,4993$ (constante de suavização do nível); $C=0,0000$ (constante de suavização da tendência) e $\mathrm{D}=0,3865$ (constante de suavização da sazonalidade). 
Utilizando os critérios de seleção descritos na Tabela 2, o modelo aditivo ajustou-se de forma mais adequada à série histórica, obtendo melhores resultados de ajuste e previsão em relação ao modelo multiplicativo e, portanto, foi o escolhido para representar o método.

Na Figura 13, podem ser observados as séries real, ajustada e prevista, modelo Holt-Winters aditivo.

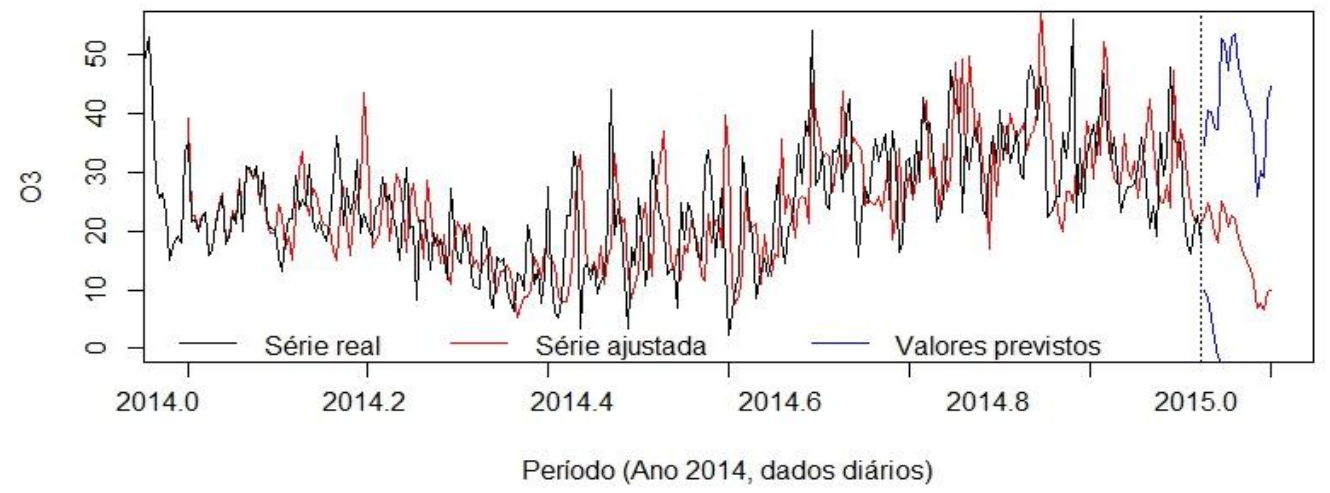

Figura 13 - Holt-Winters aditivo ajustado à variável $\mathrm{O}_{3}$.

Apesar da série apresentar sazonalidade o modelo não se ajustou adequadamente a série observada e consequentemente não produziu resultados adequados de previsão. Possivelmente uma série de dados com maior número de observações/mais extensa facilitaria para o modelo captar a sazonalidade e melhorar seu desempenho. Dentre os modelos testados o HW aditivo, obteve os piores resultados de previsão, conforme pode ser observado na tabela 6 .

\subsection{COMPARAÇÃO DAS PREVISÕES DOS MELHORES MODELOS AJUSTADOS DE CADA CLASSE}

Um dos principais objetivos da modelagem de séries temporais é fazer previsões de valores futuros. Para tanto, se faz necessário verificar a eficiência e o bom ajuste do modelo. Conforme proposto nos materiais e métodos, foram reservadas as últimas vinte observações da série observada $\left(\mathrm{O}_{3}\right)$ para comparar a qualidade das previsões dos modelos propostos dentro de cada classe correspondente.

Os critérios a serem utilizados para comparação das previsões dos modelos propostos estão descritos na tabela 2 da revisão bibliográfica. Na tabela 6, estão expostos os resultados encontrados para os modelos que melhor se ajustaram à série de dados.

Tabela 6 - Comparação dos modelos utilizando os critérios de seleção

\begin{tabular}{cccccc}
\hline Modelo & Variável & MAPE $(\%)$ & EQM & MAD & SSE \\
\hline SARIMA $(1,1,1)(0,1,1)$ & $\mathrm{O}_{3}$ & $20,26 \%$ & 34,50 & 5,87 & 651,71 \\
SARIMAX $(1,1,1[1,1,1])(0,1,1)$ & $\mathrm{O}_{3}$ & $17,3 \%$ & 28,44 & 5,33 & 635,88 \\
ALS & $\mathrm{O}_{3}$ & $23,12 \%$ & 29,88 & 6,16 & 1207,90 \\
\hline
\end{tabular}




$\begin{array}{cccccc}\text { ALB } & \mathrm{O}_{3} & 24,73 \% & 30,81 & 5,93 & 1249,3 \\ \text { HW Aditivo } & \mathrm{O}_{3} & 26,5 \% & 57,53 & 7,58 & 1387,6\end{array}$

Considerando os resultados dos critérios propostos, o modelo SARIMAX obteve melhor desempenho em todos os critérios utilizados em relação aos modelos concorrentes. Demonstrando que o ajuste e as previsões se aproximam de forma mais adequada à série observada quando se utilizam as variáveis climatológicas temperatura máxima, mínima e evaporação como exógenas aos modelos de previsão. Entretanto, cabe salientar que se faz necessário utilizar as técnicas de análise multivariada, citas nesse trabalho, para selecionar as variáveis climatológicas que contribuam significativamente sobre a variável endógena $\mathrm{O}_{3}$.

Para outras regiões é necessário que se faça novos testes/ajustes, pois a falta de dados e séries históricas mais longas dificulta a generalização/afirmativa que as variáveis selecionadas nesse trabalho (temperatura máxima, mínima e evaporação) contribuam igualmente para os poluentes do ar em estudo.

\section{CONCLUSÃO}

A pesquisa demonstra a importância do estudo conjunto de modelos estatísticos como análise multivariada e séries temporais, suas principais implicações, vantagens e desvantagens. Suas aplicações a dados de poluentes do ar e a variáveis meteorológicas, podem trazer ganhos consideráveis para essa área. Entretanto, a falta de séries históricas mais longas e completas de poluentes e também para diferentes localidades dificulta o estudo e limita a análise para a região em estudo. Para outras regiões se faz necessário reaplicar as técnicas e comparar o desempenho dos modelos.

Ao final deste trabalho, observaram-se as principais relações entre os poluentes do ar e variáveis climatológicas, para a região de Canoas-RS, com a formação de dois grupos bem definidos: a variável $\mathrm{O}_{3}$ associa-se às variáveis climatológicas Isolação, Evaporação, Temperatura Mínima e Máxima (grupo 1), e as variáveis $\mathrm{PM} 10, \mathrm{SO}_{2}$ e $\mathrm{CO}$ formam o grupo 2 e não estão diretamente sendo influenciadas pelas variáveis climáticas analisadas.

As classes de modelos de previsão utilizadas e comparadas para dados de poluição do ar, demonstraram desempenhos diferentes de previsão. Os modelos da classe de alisamento exponencial não se ajustaram adequadamente às séries em estudo. Dessa forma, não se recomenda o uso destes modelos para essas variáveis em estudo. Já os modelos da classe SARIMA e SARIMAX demonstraram desempenho satisfatório às séries em estudo e podem ser empregados para as variáveis de poluentes do ar, desde que sejam seguidos todos os pressupostos exigidos pelos modelos.

Em comparação entre as previsões dos melhores modelos encontrados para a série $O_{3}$, SARIMA $(1,1,1)(0,1,1)$ e o $\operatorname{SARIMAX}(1,1,1[1,1,1])(0,1,1)$, 0 modelo que permite a inclusão de variáveis exógenas SARIMAX demonstrou melhor desempenho de previsão. Dessa forma, percebe-se a importância do uso de variáveis meteorológicas como exógenas quando se pretende prever índices 
de poluição. Entretanto, faz-se necessário utilizar modelos multivariados para selecionar as variáveis exógenas de modo a se ter melhor eficiência de previsão.

Diante dessas análises, destaca-se a ampla possibilidade de utilização destas e de outras técnicas adequadas de análise multivariada em conjunto com séries temporais nas mais diversas áreas em que se tem por objetivo descrever um processo temporal ou quando se deseja fazer previsões, podendo ser utilizadas como ferramenta de apoio nas tomadas de decisão. Demonstrando ser uma prática simples e eficiente.

Em estudos futuros, pretende-se ajustar modelos de Previsão de Séries Temporais para o grupo 2 encontrado neste trabalho e verificar a relação de poluentes do ar e variáveis meteorológicas de uma quantidade maior de cidades.

\section{REFERÊNCIAS BIBLIOGRAFICAS}

AKAIKE, H. A Bayesian analysis of the minimum AIC procedure. Annals of the Institute of Statistical Mathematics, v. 30, p. 9-14, 1978.

A new look at the statistical model identification. IEEE Transactions on Automatic Control, v. 19, p. 716-723, 1974.

BARBOSA, C. M.; CHAVES, C. A. Um estudo sobre gerenciamento da demanda nos sistemas de planejamento e controle da energia de produção. VIII Congresso Nacional de Excelência em Gestão, de 08 a 09 de junho, São Paulo, 2012.

BARCELLOS, C.; Mudanças climáticas e ambientais e as doenças infecciosas: cenários e incertezas para o Brasil. Epidemiologia e Serviços de Saúde, v. 18, n. 3, p. 285-304, 2009.

BATISTA, A. L. F. Modelos de séries temporais e redes neurais na previsão de vazão. 2009, 79f. Dissertação de Mestrado (Mestrado em Engenharia de Sistemas) - Universidade Federal de Lavras, Lavras, 2009.

CARVALHO, V. S. B.; CAVALCANTI, P. M. P. S.; CATALDI, M.; PIMENTEL, L. C. G. Avaliação da Concentração do Ozônio e de seus precursores na RMRJ e correlação deste com variáveis meteorológicas durante o ano de 2002. In: Congresso Brasileiro de Meteorologia, XIII, 2004, Fortaleza. Anais eletrônicos. Disponível em: http://www.cbmet.org.br/cbm-files/22106a74513a8169304ab1ec402bddd658.doc. Acesso em: 15 de dezembro de 2019.

CHECHI, L.; SANCHES, F. O. Análise de uma série temporal de precipitação para Erechim (RS) e um possível método de previsão climática. Ambiência Guarapuava, v. 9, p. 43-45, 2013.

ESQUIVEL, R. M.; SENNA, V.; GOMES. G. S. S. Análise espectral singular: comparação de previsão em séries temporais. Revista do Mestrado em Administração e Desenvolvimento Empresarial, v. 16, n. 2, p. 87-101, 2012.

FELICIANO, R. A. Uma proposta de gerenciamento integrado de demanda e distribuição, utilizando sistemas de apoio à decisão (SAD) com Bussiness Intelligence (BI). 2009, 179f. Dissertação (Mestrado em Engenharia) Politécnica Universidade de São Paulo, São Paulo, 2009. 
FEPAM. Fundação Estadual de Proteção Ambiental Henrique Luiz Roessler. RS. Disponível em http://www.fepam.rs.gov.br/. Acesso em: 15 nov. 2016.

GIODA, A.; GIODA, F. R. A influência da qualidade do ar nas doenças respiratórias, Revista Ambiente e Saúde, v. 7, n. 1, p. 15-23, 2006.

IEMA. Instituto de Energia e Meio Ambiente. $1^{\circ}$ Diagnóstico da rede de monitoramento da qualidade do ar no Brasil. 2014. Disponível em: <http://www.forumclima.pr.gov.br/

arquivos/File/Rosana/Diagnostico_Qualidade_do_Ar_Ver sao_Final_Std.pdf>. Acesso em: 02 mar. 2017.

INMET. Instituto Nacional de Meteorologia. Disponível em: http://www.inmet.gov.br/. Acesso em: 15 nov. 2016.

LEITE, R. C. M.; Guimarães, E. C.; Lima, E. A. P.; Barrozo, M. A. de S.; Tavares, M. Utilização de regressão logística simples na verificação da qualidade do ar atmosférico de Uberlândia. Engenharia Sanitária e Ambiental, v. 16, n. 01, 2011.

LÍRIO, G. S. W.; SOUZA. A. M. A satisfação dos serviços pós-venda de clientes de um veículo de comunicação escrita, sob a ótica da Análise Multivariada. Revista Ciência e Natura, v. 30, n. 2, p. 21-41, 2008.

LIZZI, E. A. S. Predição do número de casos mensal dos casos de dengue por modelos de séries temporais. 2012, 131f. Dissertação (Mestrado em Saúde na Comunidade) Universidade de São Paulo (USP), Ribeirão Preto, 2012.

LÚCIO, P. S.; Silva, F. D. dos S.; Fortes, L. T. G.; Santos, L. A. R.; Ferreira, D. B.; Salvador, M. de A.; Balbino, H. T.; Sarmanho, G. F.; Dos Santos, L. S. F. C.; Lucas, E. W. M.; Barbosa, T. F.; Dias, P. L. S. Um modelo estocástico combinado de previsão sazonal para a precipitação no Brasil. Revista Brasileira de Meteorologia, v. 25, n. 1, p. 70-87, 2010.

MARTÍNEZ, R. O.; ZAMPROGNO, B. Comparação de algumas técnicas de previsão em análise de séries temporais. Revista Colombiana de Estatística, v. 26, n. 2, p. 129-157, 2003.

MONTE, E. Z.; AlBUQUERQUE, T. T. A.; REISEN, V. A. Previsão da concentração de ozônio na Região da Grande Vitória, Espírito Santo, Brasil, utilizando o modelo ARMAX- GARCH. Revista Brasileira de Meteorologia, v. 30, n. 3, 285294, 2015.

MOREIRA, D. M.; TIRABASSI, T.; MORAES, M. R.; Meteorologia e Poluição Atmosférica. Ambiente \& Sociedade. v. 11, n 1, 2008.

MORETTIN, P. A.; TOLOI, C. M. C. Análise de Séries Temporais. São Paulo: Edgard Blucher LTDA., 2004, 535 p.

. Previsão de Séries Temporais. 2. ed. São Paulo: Editora Atual Editora LTDA., 1987, 436p.

NASCIMENTO, H. H. S. Planejamento de mercado de energia elétrica no novo modelo do setor elétrico brasileiro pela aplicação de análise de componente principal como métodos de combinação de previsão. 2013, 100f, Dissertação (Mestre em Engenharia Elétrica) Universidade Federal do Mato Grosso do Sul (UFMS), Campo Grande, 2013. 
OZAKI, V. A.; ADMI, A. C. O. Modelagem estatística dos prêmios do seguro rural. Revista Política Agrícola, v. 21, n. 1, p. 60-75, 2012.

REISEN, V. A.; MOLINARES, F. A. A.; TEIXEIRA, E. C. Modelagem de Séries Temporais Sazonais na Presença de Outliers Estudo de Caso da Vazão Máxima Mensal do Rio Jucu, ES, Brasil. Revista Brasileira de Recursos Hídricos, v. 13, n. 2, 2008.

RINALDI, J. G. S.; MORABITO, S.; TACHIBANA, V. M. A importância da rapidez de atendimento em supermercados: um estudo de caso. Revista Gestão e Produção, v. 16, n. 1, p. 1-14, 2009.

SAAD, D. S.; Aplicação de técnicas estatísticas multivariadas em dados de cerâmica vermelha produzidas na região central do Rio Grande do Sul. Dissertação 166f. Programa de Pós graduação em engenharia Civil, Universidade Federal de Santa Maria (UFSM), Santa Maria, 2009.

Santos, T. C.; Reboita, M. S.; Carvalho, V. S. B. Investigação da Relação entre Variáveis Atmosféricas e a Concentração de MP10 E O3 no Estado de São Paulo. Revista Brasileira de Meteorologia, v. 33, v. 4, 631 645, 2018.

SANTOS, A. S. D. A. S.; ARISTONE, F.; KOVAČ-ANDRIĆ, E.; MATASOVIĆ , PIRES, B. J.C.; IKEFUTI, P. V. Impacto de fatores meteorológicos sobre as concentrações de ozônio modelados por análise de séries temporais e métodos estatísticos multivariados. HOLOS, Ano 33, Vol. 05, 2017. 